\title{
Antibacterial Effect of Zinc Oxide Nanoparticles on Mupirocin-Resistant Staphylococcus aureus Isolated From Nasal Carriers
}

\author{
Afsaneh Hajimohammad ${ }^{1}$, Leila Fozouni ${ }^{1 *}$ \\ ${ }^{1}$ Department of Biology, Gorgan Branch, Islamic Azad University, Gorgan, Iran
}

\author{
*Correspondence to \\ Leila Fozouni, Assistant Professor, \\ Department of Biology, Gorgan \\ Branch, Islamic Azad University, \\ Gorgan, Iran/ \\ Tel:+98-911-151-8674 \\ Fax: +98-11- 3329496 \\ Email: lili_kia@yahoo.com
}

Received September 27, 2017 Accepted April 3, 2018 Published online June 30, 2018

Please cite this article as follows: Hajimohammad A, Fozouni L. Antibacterial effect of zinc oxide nanoparticles on mupirocin-resistant Staphylococcus aureus isolated from nasal carriers. Int J Basic Sci Med. 2018;3(2):7882. doi:10.15171/ ijbms.2018.14.

\begin{abstract}
Introduction: Staphylococcus aureus is one of the main causes of hospital infections. The present study aimed to determine the antimicrobial effect of zinc oxide nanoparticles (ZnONPs) on the growth of mupirocin-resistant $S$. aureus isolates taken from hospital carriers.

Methods: The samples were taken from the anterior nasal parts of 150 hospital staff in Gorgan, using a sterile swab. Being cultured on Mannitol salt agar, the suspected colonies were identified through gram staining as well as catalase, coagulase and DNase tests. Resistance of the strains to mupirocin was examined using microdilution broth test. The antibacterial effect of ZnONPs on the mupirocin-resistant strains was also investigated using agar well diffusion method.

Results: In this study, 48 isolates (32\%) were identified as S. aureus, out of which 3 isolates $(6.2 \%)$ showed high resistance to mupirocin and 14 isolates $(29.2 \%)$ showed low resistance to this antibiotic. The results of this study revealed that ZnONPs had the highest inhibitory effect on the growth of mupirocin-resistant $S$. aureus in the density of $400 \mathrm{mg} / \mathrm{mL}$.

Conclusion: The ZnONPs used in this study had a high dose-dependent antimicrobial activity against all drug-resistant $S$. aureus strains.

Keywords: Staphylococcus aureus, Mupirocin, Zinc oxide nanoparticles, Hospital carriers
\end{abstract}

\section{Introduction}

Staphylococcus aureus is a major cause of skin and soft tissue infections, endocarditis, osteomyelitis, toxic shock syndrome (TSS), and the infections related to medical tools which could threaten human lives. ${ }^{1,2}$

Human being is the main carrier of $S$. aureus. This asymptomatic bacterium is able to settle on the skin or inside the nasal cavity of humans and animals, to the extent that about $20 \%$ of humans are permanent carriers of this bacterium. ${ }^{3}$ In some hospitals, this bacterium is more commonly found in the intensive care unit (ICU) which could be lethal. Due to increasing drug resistance, this bacterium has now turned into an important hygiene problem worldwide. ${ }^{4,5}$

After observing the first case of methicillin-resistant Staphylococcus aureus (MRSA) (1960s), mupirocin was the first topical drug used to treat MRSA skin infections in
1986. This drug is a unique antimicrobial agent in comparison with other antibiotics, which is originated from Pseudomonas fluorescens. ${ }^{6}$

Mupirocin (pseudomonic acid A) has the capacity to interact with isoleucyltRNA synthetase (IRS) and block protein synthesis. $^{7}$ Due to its short half-life after being injected and high protein connections, this drug is currently used in the topical form. Unfortunately, the current indiscriminate use of drugs has developed a relative drug resistance in this bacterium and has created resistant species of this bacterium which hinder treatment of some diseases. Therefore, it is recommended to use various combinations or antimicrobial agents such as nanoparticles to stop or control such resistance. ${ }^{8,9}$

Nanotechnology, a hotspot in science of materials, is used increasingly in researches and has applications related to human

(c) 2018 The Author(s); Published by Zabol University of Medical Sciences. This is an open-access article distributed under the terms of the Creative Commons Attribution License (http://creativecommons.org/licenses/by/4.0), which permits unrestricted use, distribution, and reproduction in any medium, provided the original work is properly cited. 
health. Many ion metal oxides are considered not only for their wide range of physical and chemical features, but also for their antibacterial activities. Zinc oxide nanoparticles (ZnONPs) yield selective toxicity and could be used as antimicrobial agents with ideal potential relative to some antibiotics. ${ }^{10,11}$ These combinations have been used as antimicrobial agents. ZnONPs act by destroying the bacterial walls and are effective in preventing antimicrobial activities in prosthesis and catheter surfaces due to the antimicrobial activities of zinc oxide as a coating material. ${ }^{12,13}$

This study aimed to evaluate and compare the antimicrobial effects of ZnONPs on the isolates of mupirocin-resistant $S$. aureus separated from the carrier staff of hospitals.

\section{Methods}

\section{Bacterial Strains}

In this study, 150 samples were isolated from 4 hospitals in Gorgan, North of Iran, during 2016-2017. Samples were taken by cotton swabs from the nose of a number of the personnel willing to participate in the study. Receiving no antibiotics over the past month and no systemic diseases or immune deficiencies were regarded as the benchmark for entering the study.

Samples were cultured on the Mannitol salt agar medium (Merck, Germany) and were incubated at $35^{\circ} \mathrm{C}$ for $24-$ 48 hours. Yellow colonies (mannitol fermenting and suspected to contain $S$. aureus) were again cultured on the same medium for further tests. S. aureus was identified through colony morphology, Gram staining, hemolysis, catalase, coagulase slide (clumping) and tube and DNase tests.

Minimum Inhibitory Concentration Determination For this purpose, we used broth microdilution method. In this method, in the 96-well microplates containing $50 \mu \mathrm{L}$ of Mueller-Hinton broth (Merck, Germany), 50 $\mu \mathrm{L}$ of the $1024 \mu \mathrm{g}$ concentration was poured in the first well and dilution was performed in all wells. The stock solutions of mupirocin were diluted in sterilized distilled water to achieve drug concentrations of 0.016 to $1024 \mu \mathrm{g} /$ $\mathrm{mL}$. Afterwards, $50 \mu \mathrm{L}$ of the bacterial suspension with the final concentration of $1.5 \times 10^{8} \mathrm{CFU} / \mathrm{mL}$ were added to each well. At the end, microplates were incubated for 24 hours at $37^{\circ} \mathrm{C}$. After incubation, the optical density (OD) of wells was read using Elisa Reader, and the first well without turbidity was regarded as minimum inhibitory concentration (MIC). Afterwards, the results of susceptibility and resistance of the strains to mupirocin were reported according to CLSI documents. ${ }^{14}$ According to the instructions of this committee and other documents, ${ }^{15}$ strains with $\mathrm{MIC} \leq 4 \mu \mathrm{g} / \mathrm{mL}$ are sensitive, the ones with $\mathrm{MIC}=8-256 \mu \mathrm{g} / \mathrm{mL}$ have low resistance and the ones with MIC $\geq 512 \mu \mathrm{g} / \mathrm{mL}$ are very resistant to mupirocin. In this method, S. aureus ATCC 25923 strain was used as the standard strain.

Antibacterial Properties of $\mathrm{ZnONPs}$

In order to prepare various concentrations of $\mathrm{ZnONPs}$, $0.8 \mathrm{~g}$ of ZnONPs powder with the size of $20 \mathrm{~nm}$ (Nanopishgaman, Iran) was added to a sterile tube containing $2 \mathrm{~mL}$ of sterile distilled water and dimethyl sulfoxide (DMSO) to reach the final concentration of 400 $\mathrm{mg} / \mathrm{mL}$ (Figure 1). The tubes were shaken for 30 minutes, and sequential concentrations of 200, 100, 50, 25 and $12.5 \mathrm{mg} / \mathrm{mL}$ were prepared in distilled water. Afterwards, microbial suspension was prepared from all mupirocinresistant strains and cultured in the form of spreadsheet on the Mueller-Hinton agar medium which contained 2\% salt. Then, wells with the diameter of $7 \mathrm{~mm}$ were drilled in the medium using sterile pipette Pasteur, and $100 \mathrm{uL}$ of $\mathrm{ZnO}$ dilutions were inoculated into the wells. One well containing distilled water was considered as the negative control. Plates were incubated at $37^{\circ} \mathrm{C}$. After 24 hours, the diameter of the inhibition zone created around each well was measured and recorded in millimeters. Zones with the diameter of over $12 \mathrm{~mm}$ were considered as susceptible to nanoparticles and the ones with the diameter of less than $10 \mathrm{~mm}$ were regarded as resistant to nanoparticles. Each stage of the test was repeated 3 times.

\section{Statistical Analysis}

To analyze the data, chi square and one-way analysis of variance (ANOVA) were performed using SPSS version 20.0. $P<0.001$ was regarded as the significance level.

\section{Results}

Staphylococcus aureus isolates were obtained from various occupational groups including technicians, service staff (total number of each group $=37$ ), nurses and physicians (total number of each group $=38$ ) who were carriers of Staphylococcus (Table1).

The strains were also studied in terms of susceptibility to mupirocin using broth microdilution method. The MIC range achieved for mupirocin in comparison with the MIC of the control strain was $\mathrm{MIC}<8 \mu \mathrm{g} / \mathrm{mL}$, and $64.6 \%$ of $S$. aureus belonged to this range of MIC. Mupirocin with the MIC of $1024 \mu \mathrm{g} / \mathrm{mL}$ showed the best inhibitory effect. Three samples $(6.2 \%)$ were in the range of MIC

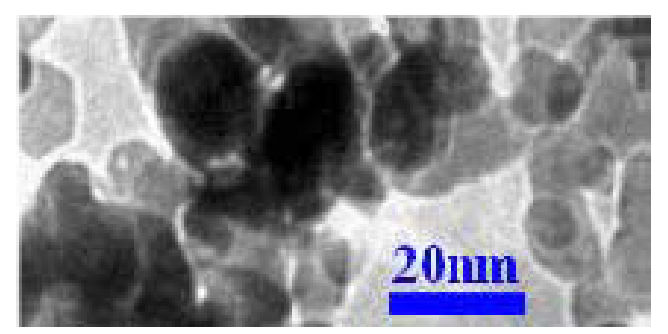

Figure 1. Zinc Oxide Nanoparticles Imaged by Transmission Electron Microscopy. 
Table 1. The Frequency of Staphylococcus aureus Strains Isolated From Nasal Carriers in Hospital

\begin{tabular}{lcc}
\hline Groups & $\begin{array}{c}\text { Absolute Abundance } \\
(\text { No.) }\end{array}$ & $\begin{array}{c}\text { Relative Abundance } \\
\mathbf{( \% )}\end{array}$ \\
\hline Service staff & 11 & 22.9 \\
Technicians & 10 & 20.8 \\
Physicians & 12 & 25 \\
Nurses & 15 & 31.3 \\
Total & 48 & 100 \\
\hline
\end{tabular}

$\geq 512 \mu \mathrm{g} / \mathrm{mL}$, and were regarded as high mupirocinresistant $S$. aureus, and $29.2 \%$ showed low resistance to this antibiotic.

The results of antibacterial effects of ZnONPs showed that there was no significant difference between $S$. aureus strains with high and low resistance to mupirocin. In this study, the best and most effective inhibitory dose of nanoparticles was $400 \mathrm{mg} / \mathrm{mL}$ (Table 2).

\section{Discussion}

Today we face antibiotic resistance that has turned to a major challenge for medical community in treating infectious diseases. ${ }^{3}$

Staphylococcus aureus is one of the most important causes of hospital infections. ${ }^{4}$ Increasing antibiotic resistance may negate consumption of mupirocin and increase the costs and duration of the treatment.

In this study, 32\% of the hospital personnel carried $S$. aureus in their noses. In medical textbooks, the amount of $S$. aureus carriers among hospital staff is reported to be $70 \%-90 \%{ }^{16}$ which is considerably more than the amount reported in the present study.

In a similar study carried out in Iran by Zeinalinia et al, ${ }^{17}$ the amount of carriers was $26.8 \%$, which was less than the amount reported in the present study.

Nagant et al isolated 1971 strains of $S$. aureus, out of which $3.6 \%$ were resistant to mupirocin. ${ }^{18}$ In the present study, $6.2 \%$ showed high resistance to mupirocin, which almost matches the results of the recent study.

In the present study and in that of Lim's which was conducted in Kualalampour, the percentage of strains with lower resistance was more than those with higher resistance. ${ }^{19}$ However, according to the study carried out by Joshi et al and the one by Chaturvedi et al in India and that of Babu et al during 2007-2008 in the United States, the strains with higher resistance were more frequent. ${ }^{20-22}$ The reason for such high resistance to mupirocin in these studies might be the indiscriminate consumption of the antibiotic or non-selection of proper anibiotic for treatment in those hospitals.

In the study carried out by Saderi on 94 S. aureus isolates from 4 hospitals affiliated to the University of Tehran using PCR, 6 isolates were found to be resistant to mupirocin. Five isolates had low resistance and 1 showed high resistance. ${ }^{23}$
Table 2. Diameter of Inhibition Zone Related to the Effect of Zinc Oxide Nanoparticles on the Growth of Mupirocin-resistant Staphylococcus aureus Isolates

\begin{tabular}{lll}
\hline \multicolumn{3}{c}{ Inhibition Zone Diameter $(\mathbf{m m})$} \\
\hline ZnO Concentrations $(\mathbf{m g} / \mathbf{m L})$ & Mean & SD \\
\hline 400 & 14.1 & 4.00 \\
200 & 10.7 & 3.7 \\
100 & 7.00 & 0.2 \\
50 & 7.00 & 0 \\
25 & 7.00 & 0 \\
12.5 & 7.00 & 0 \\
\hline
\end{tabular}

$P$ value $<0.001$

In a study carried out on $S$. aureus nasal carriers in the staff of a hospital in Sweden, the highest amount of this bacterium was reported to be in male staff (33.3\%). ${ }^{24}$ This is in contrary to the present study, where the highest frequency was seen in female staff (52\%).

In another study conducted by Karamstaji et al, the highest frequency of $S$. aureus was found in the radiology and laboratory sections $(26.66 \%)$ and the lowest frequency was found in the neonatal ward. ${ }^{25}$ In the present study, the most and the least frequent carriers of $S$. aureus were the groups of nurses and technicians with $31.3 \%$ and $20.8 \%$, respectively.

Various studies have revealed that making use of mupirocin antibiotic can considerably reduce infections caused by staphylococci. However, increasing resistance may negate consumption of this antibiotic and increase the costs and duration of the treatment.

One of the promising strategies for confronting bacterial resistance is to use metal nanoparticles. In this study, the antimicrobial activity of various concentrations of ZnONPs was studied on S. aureus resistant to mupirocin. In the present study, the antibacterial properties of zinc oxide nanoparticles were shown. Seil et al in the United States synthesized $\mathrm{ZnONPs}$ and studied their antibacterial properties on S. aureus, and showed that zinc oxide owns antibacterial effects. ${ }^{26}$

Zhang et al studied the antimicrobial effects of variable concentrations of $\mathrm{ZnONPs}$ with different sizes on Escherichia coli. They found that the antimicrobial effect increased as the concentration of the nanoparticle was raised independent of their sizes. ${ }^{27}$ Likewise, in the present study, the best inhibitory effect was seen when the concentration of the nanoparticle was increased to 400 $\mathrm{mg} / \mathrm{mL}$.

Li et al studied the antimicrobial effects of $\mathrm{ZnONPs}$ on the coat of polyvinyl chloride film on S. aureus and E. coli. ${ }^{28}$ They reported that ZnONPs better affected Gram-positive bacteria than Gram-negative ones, and the reason for this was the difference between the membrane structure of gram-positive and gram-negative bacteria as well as the difference in the thickness of their peptidoglycan. Grampositive bacteria such as $S$. aureus have multilayer and 
thick peptidoglycan, while the peptidoglycan is thiner in Gram-negative bacteria. However, their exterior membrane has lipopolysaccharide which is less permeable to certain antibiotics and antimicrobial agents.

\section{Conclusion}

The results revealed that $S$. aureus exists in the nose of hospital staff and therefore can possibly be transferred to others especially the hospitalized patients. Thus, in order to prevent outbreaks of infections, it is important that hospital staff consider washing hands as well as applying specific methods for isolating and making use of topical antimicrobial agents in order to reduce colonization in the nose and prevent further infections in clinical settings. Our results showed that $\mathrm{ZnONPs}$ have antibacterial effects, and their antimicrobial effect increases upon increasing the concentration of the nanoparticles.

\section{Author's Contribution}

LF contributed to the study concept, and designed, supervised, and edited the final manuscript. $\mathrm{AH}$ performed sample collection and laboratory examinations and interpreted the data. All authors discussed the results and implications and provided their comments during all the stages.

\section{Competing Interests}

None declared.

\section{Ethical Approval}

Not applicable.

\section{Acknowledgments}

This work was supported by the Research Council of the Islamic Azad University, Gorgan Branch, Iran. We would like to thank the hospital staff in the city of Gorgan.

\section{References}

1. Thapaliya D, Forshey BM, Kadariya J, et al. Prevalence and molecular characterization of Staphylococcus aureus in commercially available meat over a one-year period in Iowa, USA. Food Microbiol. 2017;65:122-129. doi:10.1016/j. fm.2017.01.015

2. Jans C, Merz A, Johler S, et al. East and West African milk products are reservoirs for human and livestock-associated Staphylococcus aureus. Food Microbiol. 2017;65:64-73. doi:10.1016/j.fm.2017.01.017

3. Tong SY, Davis JS, Eichenberger E, Holland TL, Fowler VG Jr. Staphylococcus aureus infections: epidemiology, pathophysiology, clinical manifestations, and management. Clin Microbiol Rev. 2015;28(3):603-661. doi:10.1128/ cmr.00134-14

4. van Belkum A, Verkaik NJ, de VogelCP, et al. Reclassification of Staphylococcus aureus nasal carriage types. J Infect Dis. 2009;199(12):1820-1826. doi:10.1086/599119

5. Kluytmans J, van Belkum A, Verbrugh H. Nasal carriage of Staphylococcus aureus: epidemiology, underlying mechanisms, and associated risks. Clin Microbiol Rev. 1997;10(3):505-520.

6. Kong EF, Johnson JK, Jabra-Rizk MA. CommunityAssociated Methicillin-Resistant Staphylococcus aureus: An Enemy amidst Us. PLoS Pathog. 2016;12(10):e1005837. doi:10.1371/journal.ppat.1005837

7. Cern A, Michael-Gayego A, Bavli Y, et al. Nano-mupirocin: enabling the parenteral activity of mupirocin. Eur J Nanomed. 2016;8(3):139-149. doi:10.1515/ejnm-20160006

8. Casewell MW, Hill RL. In-vitro activity of mupirocin ('pseudomonic acid') against clinical isolates of Staphylococcus aureus. J Antimicrob Chemother. 1985;15(5):523-531. doi:10.1093/jac/15.5.523

9. Mahon CR, Lehman DC, Manuselis G Jr. Textbook of Diagnostic microbiology. 3rd ed. Saunders; 2007:369.

10. Sirelkhatim A, Mahmud S, Seeni A, et al. Review on Zinc Oxide Nanoparticles: Antibacterial Activity and Toxicity Mechanism. Nano-micro lett. 2015;7(3):219-242. doi:10.1007/s40820-015-0040-x

11. Jones N, Ray B, Ranjit KT, Manna AC. Antibacterial activity of $\mathrm{ZnO}$ nanoparticle suspensions on a broad spectrum of microorganisms. FEMS Microbiol Lett. 2008;279(1):71-76. doi:10.1111/j.1574-6968.2007.01012.x

12. Wang C, Liu LL, Zhang AT, Xie P, Lu JJ, Zou XT. Antibacterial effects of zinc oxide nanoparticles on Escherichia coli K88. Afr J Biotechnol. 2012;11(44):10248-10254. doi:10.5897/ AJB11.3703

13. Gao PX, Ding Y, Mai W, Hughes WL, Lao C, Wang ZL. Conversion of zinc oxide nanobelts into superlatticestructured nanohelices. Science. 2005;309(5741):17001704. doi:10.1126/science.1116495

14. Clinical and Laboratory Standards Institute (CLSI). Performance standards for antimicrobial susceptibility testing: M100-S17. Clinical and Laboratory Standards; 2008;26:1-182.

15. de Oliveira NE, Cavalcanti ED, Laport MS, Bastos Mdo C, Giambiagi-deMarval M. Constitutive expression of the ileS-2 gene responsible for high-level mupirocin resistance in Staphylococcus aureus. J Med Microbiol. 2009;58(Pt 12):1582-1584. doi:10.1099/jmm.0.013912-0

16. Pillie M, Yod-Ar Q, Michel P. Staphylococcus aureus. Mandell, Douglas, Bennett, eds. Principles and Practice of Infectious Diseases. Philadelphia: Elsevier; 2005:23212352.

17. Zeinalinia N, Pourmand MR, Ghne M, Afrough P. The frequency of Staphylococcus aureus infection among the staff of the hospitals affiliated to Tehran University of Medical Sciences .Journal of Hospital. 2011;10(1):71-76. [Persian].

18. Nagant C, Deplano A, Nonhoff C, et al. Low prevalence of mupirocin resistance in Belgian Staphylococcus aureus isolates collected during a 10 year nationwide surveillance. J Antimicrob Chemother. 2016;71(1):266-267. doi:10.1093/ jac/dkv286

19. Lim KT, Hanifah YA, Mohd Yusof MY, Thong KL. Prevalence of mupirocin resistance in methicillin-resistant Staphylococcus aureus strains isolated from a Malaysian hospital. Jpn J Infect Dis. 2010;63(4):286-289.

20. Joshi PR, Acharya M, Aryal R, et al. Emergence of staphylococcal cassette chromosome mec type I with 
high-level mupirocin resistance among methicillinresistant Staphylococcus aureus. Asian Pac J Trop Biomed. 2017;7(3):193-197. doi:10.1016/j.apjtb.2016.12.002

21. Chaturvedi P, Singh AK, Singh AK, Shukla S, Agarwal L. Prevalence of mupirocin resistant Staphylococcus aureus isolates among patients admitted to a tertiary care hospital. N Am J Med Sci. 2014;6(8):403-407. doi:10.4103/19472714.139293

22. Babu T, Rekasius V, Parada JP, Schreckenberger P, Challapalli M. Mupirocin resistance among methicillinresistant Staphylococcus aureus-colonized patients at admission to a tertiary care medical center. J Clin Microbiol. 2009;47(7):2279-2280. doi:10.1128/jcm.0183408

23. Saderi H, Owlia P, Habibi M. Detection of Resistance to Mupirocin in Staphylococcus aureus Strains Isolated from Patients in Four University Hospitals of Tehran by Polymerase Chain Reaction (PCR) Method. Daneshvar Medicine. 2009;16(78):31-38. [Persian].

24. Tammelin A, Klotz F, Hambraeus A, Stahle E, Ransjo U. Nasal and hand carriage of Staphylococcus aureus in staff at a department for thoracic and cardiovascular surgery: endogenous or exogenous source? Infect Control Hosp Epidemiol. 2003;24(9):686-689. doi:10.1086/502277

25. Karamstaji A, Moradi N, Bushehri A, et al. Nasal carrier rates and antibiogrm pattern of Staphylococcus aureus strains isolated from hospital staff in teaching hospitals in Bandar Abbas. Bimonthly Journal of Hormozgan University of Medical Sciences.2008:12(2)95-101: [Persian].

26. Seil JT, Webster TJ. Reduced Staphylococcus aureus proliferation and biofilm formation on zinc oxide nanoparticle PVC composite surfaces. Acta Biomater. 2011;7(6):2579-2584. doi:10.1016/j.actbio.2011.03.018

27. Zhang L, Jiang Y, Ding Y, Povey M, York D. Investigation into the antibacterial behaviour of suspensions of $\mathrm{ZnO}$ nanoparticles ( $\mathrm{ZnO}$ nanofluids). J Nanopart Res. 2007;9(3):479-489. doi:10.1007/s11051-006-9150-1

28. Li H, Li F, Wang L, et al. Effect of nano-packing on preservation quality of Chinese jujube (Ziziphus jujuba Mill. var. inermis (Bunge) Rehd). Food Chem. 2009;114(2):547-552. doi:10.1016/j.foodchem.2008.09.085 